\title{
Review of Experimental Work in Biomimetic Foils
}

\author{
Michael S. Triantafyllou, Alexandra H. Techet, and Franz S. Hover, Associate Member, IEEE
}

\begin{abstract}
Significant progress has been made in understanding some of the basic mechanisms of force production and flow manipulation in oscillating foils for underwater use. Biomimetic observations, however, show that there is a lot more to be learned, since many of the functions and details of fish fins remain unexplored.

This review focuses primarily on experimental studies on some of the, at least partially understood, mechanisms, which include 1) the formation of streets of vortices around and behind two- and three-dimensional propulsive oscillating foils; 2) the formation of vortical structures around and behind two- and three-dimensional foils used for maneuvering, hovering, or fast-starting; 3 ) the formation of leading-edge vortices in flapping foils, under steady flapping or transient conditions; 4) the interaction of foils with oncoming, externally generated vorticity; multiple foils, or foils operating near a body or wall.
\end{abstract}

Index Terms-Biomimetics, fish swimming, flapping foil propulsion.

\section{OVERVIEW OF LITERATURE}

B IOMIMETIC studies and observations from fish and cetaceans have provided a wealth of information on the kinematics, i.e., how these animals employ their flapping tails and several fins to produce propulsive and maneuvering forces (see reviews in [123] and [27]). Recent work with live animals has provided important information on the resulting flow structures [113], [3], [85], [135], [20], [21], [69].

The fluid mechanics and force mechanics of foils have been investigated with the goal of understanding the principles of this different paradigm of propulsion and maneuvering, so as to apply it to enhance existing technology. The tails of some of the fastest swimming animals closely resemble high aspect ratio foils. As a result, flapping foils have been studied extensively using theoretical and numerical techniques [72], [137], [138], [74], [13], [51], [83], [114], [115], [94], [95], and experimentally [109], [17], [68], [6], [98].

\section{BASIC PARAMETERS AND DEFINITIONS}

In a foil with maximum chord length $c$ and maximum span $s$, moving at steady speed $U$, and at an angle of attack $\alpha$, the parameters of relevance are (a) the geometric shape (rectangular, delta-shaped, etc.); (b) the aspect ratio (AR), defined to be equal to the ratio of an average span over an average chord; (c) the angle of attack $\alpha$; and (d) the Reynolds number $\operatorname{Re}=\mathrm{Uc} / \nu$, where $\nu$ is the kinematic viscosity of the fluid.

Manuscript received February 20, 2003. This work was supported by the Office of Naval Research.

The authors are with the Department of Ocean Engineering, Massachusetts Institute of Technology, Cambridge, MA 02139 USA (e-mail: mistetri@mit.edu; ahtechet@mit.edu; hover@mit.edu).

Digital Object Identifier 10.1109/JOE.2004.833216
The forces are classified as lift, the component perpendicular to the velocity $U$, and drag, the component parallel to $U$.

In unsteadily moving foils, we must first parametrize the motion of the foil. For a foil of chord $c$, moving forward at average velocity $U$, and oscillating harmonically with a linear (heave) motion $\mathrm{h}(\mathrm{t})$, transversely to the velocity $U$, and an angular (pitch) motion $\theta(\mathrm{t})$

$$
\begin{aligned}
& \mathrm{h}(\mathrm{t})=\mathrm{h}_{\mathrm{o}} \sin (2 \pi \mathrm{ft}) \\
& \theta(\mathrm{t})=\theta_{\mathrm{m}}+\theta_{\mathrm{o}} \sin (2 \pi \mathrm{ft}+\psi)
\end{aligned}
$$

where $\psi$ is the phase angle between heave and pitch, $\mathrm{h}_{\mathrm{o}}$ the heave amplitude, $\theta_{\mathrm{o}}$ the pitch amplitude, $\theta_{\mathrm{m}}$ the average pitch angle, and $\omega=2 \pi \mathrm{f}$ the frequency of oscillation, we can define the following nondimensional parameters, in addition to those for a steadily moving foil:

1) heave to chord ratio $h^{*}=h / c$;

2) maximum unsteady angle of attack $\alpha_{\max }$;

3) reduced frequency $\mathrm{k}=\mathrm{fc} / \mathrm{U}$;

4) Strouhal number, defined as $\mathrm{St}=\mathrm{Af} / \mathrm{U}$, where $A$ is the width of the wake of the foil;

5) mean angle of attack, which is equal to $\theta_{\mathrm{m}}$.

The Strouhal number is often approximated by taking $A=2 h_{o}$, i.e.,

$$
\mathrm{St}=\mathrm{Af} / \mathrm{U}=2 \mathrm{~h}_{\mathrm{o}} / \mathrm{U} .
$$

The maximum angle of attack is defined as the maximum value of the angle $\alpha(\mathrm{t})-\theta_{\mathrm{m}}$, where $\alpha(\mathrm{t})$ is defined as

$$
\tan (\alpha(\mathrm{t})+\theta(\mathrm{t}))=(\mathrm{dh} / \mathrm{dt}) / \mathrm{U} .
$$

It should be noted that, with the approximation that $\mathrm{A}=2 \mathrm{~h}_{\mathrm{o}}, \mathrm{St}=2 \mathrm{kh}^{*}$. Although "St" and $k$ can be used interchangeably, they are both useful; because St controls unsteady phenomena in the wake, while $k$ provides a measure of unsteadiness with respect to the size of the foil. High force production and efficient thrust development is often achieved when the motion of the foil is comparable to its chord; then $\mathrm{k}$ and St are of the same order of magnitude.

The instantaneous force $F$ can be decomposed into a transverse component $Z$, and an axial component $X$, with respect to the steady velocity $U$. The transverse force may contain a steady component, which can serve, as in steadily moving foils, to provide a constant lift force. The instantaneous axial force may be positive (thrust) or negative (drag); it typically contains a steady (average) component, $\mathrm{X}_{\mathrm{m}}$, which can be used to propel a body. When propulsion is the goal, a propulsive efficiency $\eta$ is defined as

$$
\eta=\mathrm{X}_{\mathrm{m}} \mathrm{U} / \mathrm{P}_{\mathrm{m}}
$$


where $\mathrm{P}_{m}$ is the time-averaged power needed to flap the foil. It must be noted that the force $\mathrm{X}_{\mathrm{m}}$ is the net average axial force acting on the foil; to employ terms from inviscid theory, it is the difference between the thrust developed through inviscid mechanisms by the motion of the foil, and the drag due to the viscous action of fluid particles. Drag and thrust are inseparable in reality, though, and $\mathrm{X}_{\mathrm{m}}$ is the net force that one would measure in the laboratory.

Inviscid theory [72] predicts efficiency close to $100 \%$ under light thrust conditions and for low frequency of oscillation. This fails to take into account the effect of drag, which would cause the net force to become negative when the thrust force is small. Nonetheless, as described in the following sections, propulsive efficiency under proper parametric conditions can be high for foils developing substantial net thrust.

\section{STEADILY OR QUASI-STEADILY OPERATING FOILS}

Extensive work has been performed in the aeronautics literature on enhancing lift in foils operating under steady or quasisteady conditions, and reducing drag [64].

Streamlining the foil is a first and obvious step. Aspect ratio is the principal parameter affecting lift production in a streamlined foil. High aspect ratio wings provide high lift capacity, comparable to that predicted for a two-dimensional foil, with small associated induced-drag coefficient. To reduce the effect of tip vortices, end appendages have been proposed, which have an effect similar to end walls - to a certain degree. In practice, it has been found that end plates are useful only for high lift coefficient, above 0.3. Trailing vanes, winglets, and tip sails, are good for improving performance of existing wings, but a to-be-designed wing can always be made as good without them, by proper aspect ratio choice.

Various means for reducing or eliminating stall, and for improving lift production of quasi-steadily operating wings have been proposed, some of them employing some unsteady excitation [75], [136]. Trapped vortices above the suction side of the wing have been tested, such as the "Kasper wing" [16], [15], [134], [65]. The stabilization of the vortex on the wing presents a major problem, since spanwise blowing — or suction — may be required to ensure that the vortex is not entrained or that the flow does not separate as vortices of opposite sign are formed, entraining the attached vortex away from the wing.

Fins are used for stability purposes. The tuna, for example, has retractable and nonretractable fins; the latter are needed to control the position of the aerodynamic center, i.e., for stability of motion. Webb [148], [149] reviews issues of fish stability.

\section{FlapPing FoILS IN BiRdS Versus Fish}

Extensive work on flying and swimming animals has identified some basic mechanisms employed through unsteadily moving foils to produce the forces needed for propulsion and maneuvering of birds and fish. The need for large lift forces in insects has forced them to employ unsteady lift-enhancing mechanisms mentioned in the previous section. Dickinson [19] classifies them as delayed stall, rotational circulation, and wake capture. Delayed stall [77], [88], [89], [32], [43], [18], the generation of large leading-edge vorticity, possibly stabilized

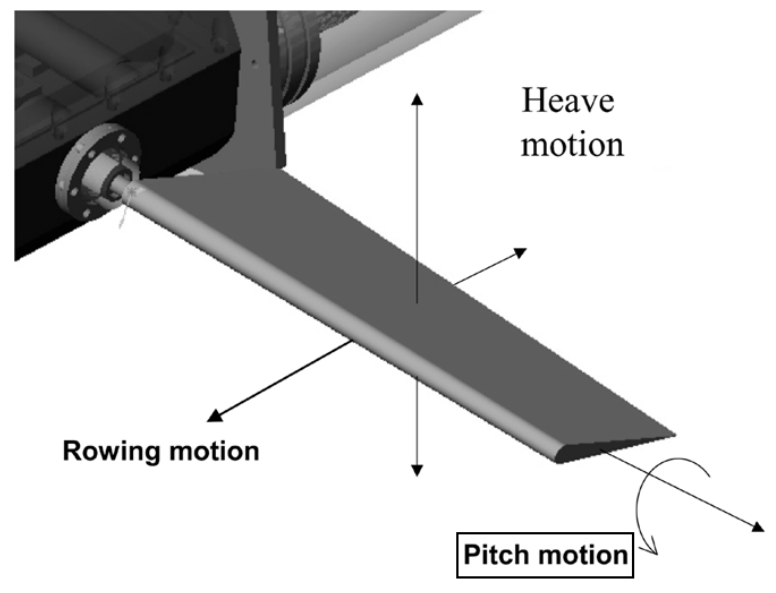

Fig. 1. Motion definitions.

by spanwise flow through the core of the leading edge vortex, is well established [81], [23], [99], [18]. Rotational velocity is also well known to enhance lift if properly timed [88], [89]. Wake capture means the interaction of the foil with previously shed vortices, especially if energy is recovered from them.

Swimming animals employ some of the same mechanisms with flying animals to produce forces, but the circumstances of swimming differ drastically from those of flying: Whereas a primary goal in flying is the continuous production of steady lift, to balance the large body weight within a medium with small density, the major goal in fish swimming is to minimize drag forces within a medium a thousand times more dense than air-the generation of steady lift to support the (small) net weight is of secondary or no importance at all. Fins and foils in water are used for continuous production of thrust or bursts of short-duration forces for maneuvering; birds must continuously support their weight, in addition to any other function. A comparison of the pleated wings of some insects [61] with the perfectly streamlined fins of most fish and cetaceans points to fundamental differences in function and operational range (Reynolds number) between fish and birds.

As already stated, there are similar mechanisms at work in fish and birds.

1) The formation of leading edge vortices ("delayed stall").

2) Influence of shed vorticity through the stable formation of Kármán streets, or interconnected patterns in faststarting foils. What is termed "wake capture" in Dickinson [19] is a form of shed vortex-foil interaction.

3) Effective angle of attack and angular velocity ("rotational circulation").

These mechanisms exist also in fish [3], [85], [135], but not to the pronounced degree exhibited in insects, for example. In Anderson et al. [6] a mild leading edge vortex (LEV) is found to benefit efficiency, but a large LEV leads to very low efficiency and high drag.

The ingenious description of lift production through the "Weis-Fogh" mechanism [133], [72] had to be revised by the development of leading edge vortices, to account for a discrepancy by a factor of three in lift generation [82]. Hence, LEVs can be a very important factor in lift development. Work with 


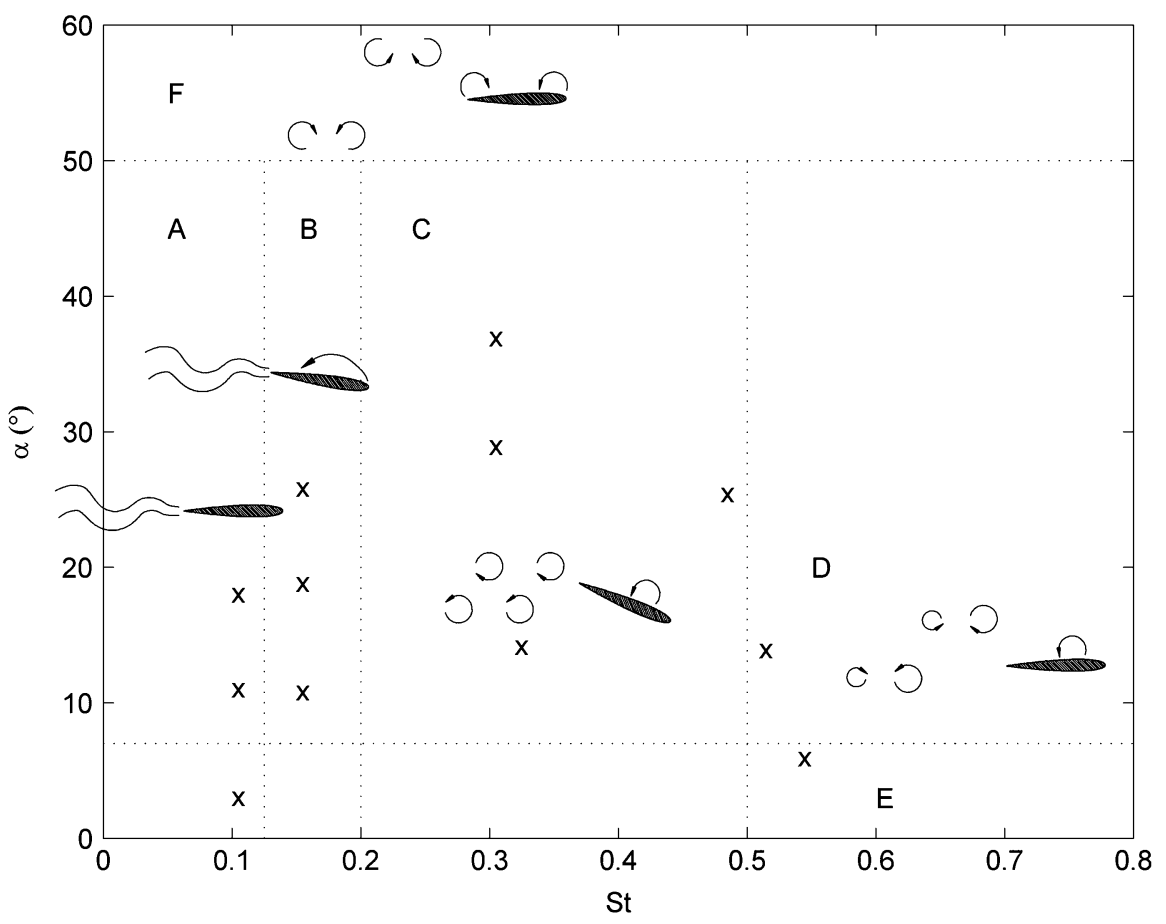

Fig. 2. Wake patterns as function of the Strouhal number and angle of attack for ho/c $=1$. Points mark the location of experiments reported in [6].

insects and small flying animals shows that LEVs, in conjunction with potential flow mechanisms, such as the Weis-Fogh mechanism, can be used to generate large lift forces. There is a penalty, though, when large LEVs develop, because there are large separation-like pressure gradients which result in large drag and lift forces. Investigation of the use of the Weis-Fogh mechanism for applications, as suggested by Furber and Ffowcs Williams [35], resulted in relatively low efficiency [121].

Measurements of forces and power in flapping foils [6], [98] show that for optimal parametric combinations the drag on a flapping foil is very close to the drag on a steadily towed foil at zero angle of attack, resulting in high efficiency. In contrast, insects, which must produce very high lift forces at low or zero forward speed, generate also high drag [25].

The physical mechanisms of force production in unsteadily flapping foils have been elucidated for flying animals, because of the large motions required to produce large lift. The particular needs of operation underwater, i.e., low drag and high efficiency at high Reynolds number, result in important differences from foils used for flying.

\section{A Review of Basic Mechanisms}

\section{A. Steadily Oscillating Two-Dimensional Foils}

The idea of forming spatially periodic patterns of vortices behind flapping foils has come out "naturally" out of the early work by Lighthill [72] and $\mathrm{Wu}$ [137]. More recent work has studied the effect that different patterns of vortices have on the forces and efficiency of foils. Triantafyllou et al. [119] performed a stability analysis of the wake to find that there are nondimensional frequencies (Strouhal numbers, as defined in Section II) for vortex formation, which are optimal for energy minimization. Data from flapping foils and swimming fish and cetaceans show that they have preferred nondimensional frequencies close to those for optimal efficiency [120], [26], [50], [68], [101], [86], [145], [146], [154], [156].

For two-dimensional foils, and high aspect ratio foils away from the ends, a planar cut in the wake shows that two vortices per cycle are the optimal pattern, resulting in the formation of a "reverse Kármán street"; more than two vortices may form [62], [68], resulting in degradation of thrust generation and propulsive performance [62], [6].

Pitching-only foils [62], heaving-only foils [50], and heaving and pitching foils [109], [6] exhibit reverse Kármán streets. For large Strouhal number, heaving only foils exhibit an instability whereby the Kármán street is developed at an angle with respect to the oncoming velocity, resulting in steady lift. There is no preferred direction, i.e., the street may be inclined with a positive or negative angle, depending on the initial conditions, while small disturbances can cause random switching in direction.

As found in [42], delta wings exhibit no dependence on the reduced frequency until large angles of attack are used, because the vortices forming from the sides remain attached and are convected downstream through a helical motion. Hence there is no characteristic time scale. In contrast, high aspect ratio foils that form leading edge and trailing edge vortices depend strongly on reduced frequency.

Fig. 2 [6] summarizes the observational data on the flow around two-dimensional flapping foils as function of the angle of attack and the Strouhal number. The Strouhal number is defined as $\mathrm{St}=f A / U$, where $f$ denotes the frequency of foil oscillation, $A$ denotes the characteristic width of the created jet flow, and $U$ is the speed of the foil.

We distinguish several regions: In regions A and B $(\mathrm{St}<0.2)$ the wake does not roll up into discrete vortices; in region B a very weak leading edge vortex appears for $a_{\max }>30^{\circ}$, but 


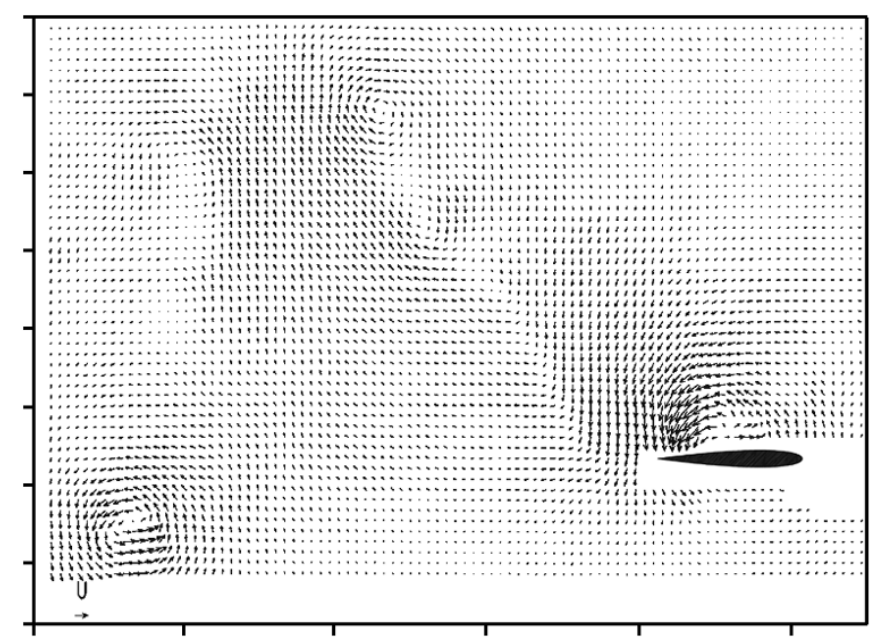

Fig. 3. DPIV velocity data for the foil at its minimum heave position, and $\mathrm{St}=0.45, \mathrm{~A} / \mathrm{c}=1, \theta=30^{\circ}, \alpha_{\max }=13.3^{\circ}, \psi=90^{\circ}[6]$.

the wake retains its wavy form. For angles of attack larger than about $50^{\circ}$ a "piston" mode appears where leading and trailing edge vortices form and roll up in the wake to form four vortices per cycle. In region $\mathrm{E}$, for angles of attack smaller than $5^{\circ}$, the wake does not form distinct patterns. Region $\mathrm{C}$, contained in the limits: $7^{\circ}<a_{\max }<50^{\circ}$ and $0.2<\mathrm{St}<0.5$, is characterized by the formation of a clear reverse Kármán street. A leading edge vortex forms for angles of attack larger than about $10^{\circ}$, increasing in strength with increasing angle of attack, which is amalgamated with trailing edge vortices to form two vortices per cycle. Region D (St > 0.5) is characterized by the formation of leading edge vortices, which interact with trailing edge vorticity to form four vortices per cycle. Data for lower $h_{\mathrm{o}} / \mathrm{c}$ show nearly identical trends as far as the wake form is concerned; the formation of a leading edge vortex depends on $\mathrm{h}_{\mathrm{o}} / \mathrm{c}$, however.

The presence of a leading edge vortex is strongly influenced by the angle of attack. In region C, for St between 0.2 and 0.5, strong thrust develops from a reverse Kármán street, accompanied by up to a moderately strong leading edge vortex. Region $\mathrm{C}$ contains the region of optimal efficiency found in the force experiments. In region $\mathrm{D}$, for St larger than 0.5 , strong thrust develops accompanied by the formation of two vortices per half-cycle, which have opposite circulation and, in general, different strength. Regions A and B are characterized by low or negative thrust, and a wavy wake with no distinct vortex formation; the leading edge vortex is very weak. In region $\mathrm{E}$, for very small angles of attack, very small or negative thrust develops.

Foils oscillating around a steady angle of attack produce asymmetric wakes, sine they generate a steady lift force [32], [33]. The wake may be inclined with respect to the oncoming flow, and/or contain larger eddies on one side of the wake, and/or a larger number of vortices on one side of the wake versus the other side. In a hovering mode, i.e., at zero oncoming speed, this allows to vector the force produced [33].

In a study on the efficiency of flapping foils [6], the LEV slightly augmented propulsive efficiency when its circulation is mild, but caused the performance to deteriorate when it grew in strength. LEV can merge with trailing edge vortices to produce a reverse Kármán street. Fig. 3 shows a mild leading edge vortex

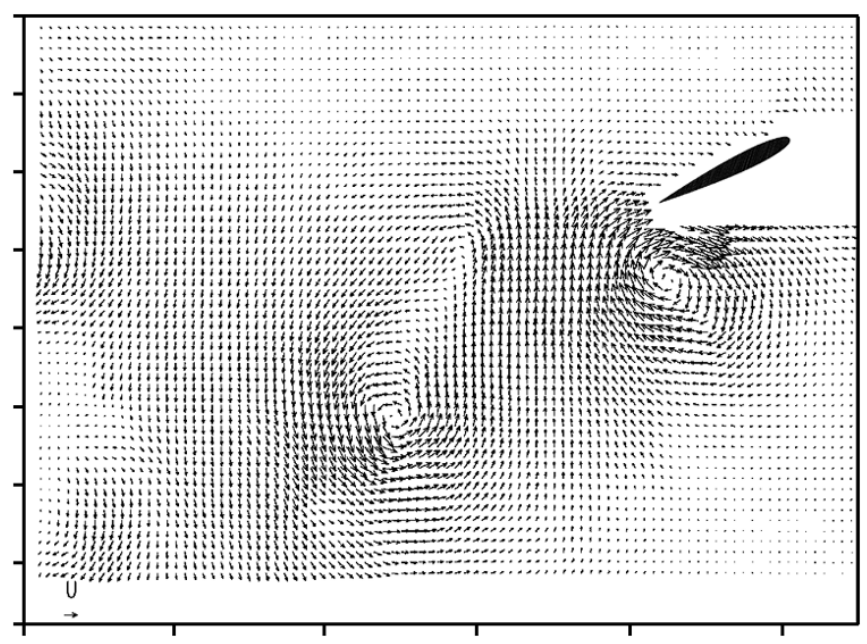

Fig. 4. DPIV velocity data for the foil at its maximum heave position, and $\mathrm{St}=0.30, \mathrm{~A} / \mathrm{c}=1, \theta=30^{\circ}, \alpha_{\max }=52.7^{\circ}, \psi=90^{\circ}[6]$.

forming on a flapping foil; behind it a reverse Kármán street forms (vortices at the top and center and bottom left). Fig. 4 shows a strong leading edge vortex, which has been shed and has reached the trailing edge of a flapping foil. Again, a reverse Kármán street forms back in the wake, consisting primarily of leading edge vorticity.

In inviscid hydrodynamic theory, the Kutta condition, i.e., the imposition of the following condition: the velocity leaves tangentially from both sides of the foil at the (presumed sharp) trailing edge, with continuous pressure across the edge. This is fundamental to deriving the forces and flow patterns around a steadily or unsteadily moving foil and although the Kutta condition is an artifact of inviscid theory, it adds to our physical understanding; hence it is interesting to consider whether it is valid in an unsteadily moving foil as well.

Evidence of apparent failure of the Kutta condition at large frequencies led to a number of investigations, which concluded that the Kutta condition is valid if one takes into account possible vortices forming close to the trailing edge [87], [108], [93].

A basic question is whether a sinusoidal motion constitutes optimal kinematics for a flapping foil, or if a different motion would provide better efficiency. Koochesfahani [64] studied various deviations from a purely pitching oscillation of a foil and found that the purely sinusoidal motion produces a clean reverse Karman street, whereas any other motion produces additional vortices per cycle. Since a reverse Karman street is found to require minimal energy, this shows that a purely oscillatory pitching motion is optimal. For a heaving, and a heaving and pitching motion, Hover et al. [155] found that the optimal kinematics in terms of propulsive efficiency are not purely sinusoidal heave and pitch motions; instead, for maximum efficiency, a multifrequency heave motion was used that, in combination with a sinusoidal pitch motion, produced a purely harmonic angle of attack.

\section{B. Steadily Oscillating Three-Dimensional Foils}

In three-dimensional foils, the vortical patterns must connect with each other and with the foil producing them. The idea 


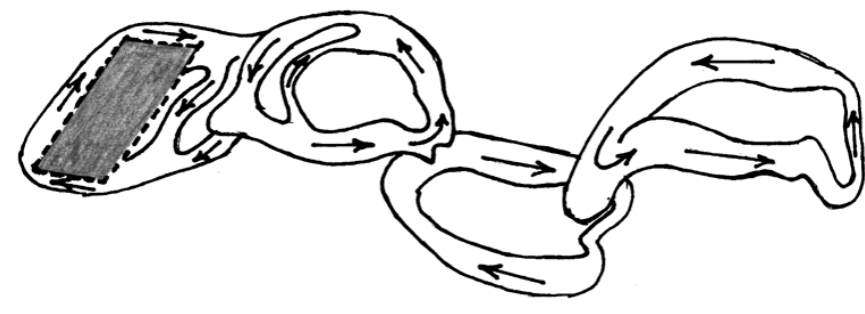

Fig. 5. Vortical patterns in a rectangular foil with aspect ratio 3, oscillating at Strouhal number 0.18 (from [150]). The patterns in the flow are isocontours of the $\lambda_{2}$ eigenvalue [153]

of a chain of alternately inclined-with respect to the direction of motion-interconnected vorticity rings, as sketched by Lighthill [72] — provides a consistent qualitative picture for the flow structure behind oscillating foils. Recent work [20], [21], [69] shows the formation of ring-like vortical structures by fish fins.

Detailed flow visualization in flapping foils provides a more complex picture [31], [33], [45]. The overall picture in three-dimensional wings is a "curious mixture of two-dimensional and three-dimensional vortex developments" [33]. The presence of leading edge vortices further complicates the connection of the vortical structures.

Fig. 5 is a sketch based on numerical simulations (DNS) by Guglielmini [150] at $\operatorname{Re}=160$, showing the vortical patterns behind a rectangular foil with $\mathrm{AR}=3$ at moderate Strouhal number. The patterns resemble irregular rings, or "pancakes," concatenated as expected by the circulation laws. This picture is in agreement with experimental results by Elllenrieder et al. [140]. The rings are often not simple, i.e., intermediate interconnections appear as in the first two rings behind the foil shown in Fig. 5, which may become weak and invisible at later stages ( $\mathrm{St}$ lower than 0.2), or may develop into large, separate branches for higher St.

The performance of three-dimensional foils depends on the reduced frequency (or, equivalently, the Strouhal number), the foil shape and aspect ratio, and the angle of attack. The effect of the aspect ratio on the forces is reduced as the reduced frequency increases, because the tip vortices are of alternating sign, hence the induced velocities are significantly reduced. This observation, first made by Karpouzian et al. [51], was also reported in the numerical study of Cheng et al. [14]. Recent detailed data on three-dimensional foils [80] show little degradation of propulsive performance for moderate aspect ratio foils, compared with the results for two-dimensional foils.

For high Strouhal number and angle of attack, it is known from two-dimensional foils that four vortices per cycle form. As shown in Fig. 6 for the same rectangular foil of Fig. 5 (aspect ratio 3 ) and higher $S t=0.35$, the vortical patterns evolve in a significantly different way: Two distinct branches appear, drifting away from each other. The interconnections are not simple, but distinct ring-like structures form, which in a vertical planar cut, near the center-plane, would appear as four major vortices per cycle.

Scherer [109] reports efficiency in rectangular, moderate aspect ratio wings of up to 70\%. Lai et al. [67] report efficiencies up to about $75 \%$ (with scatter) for a flapping rectangular

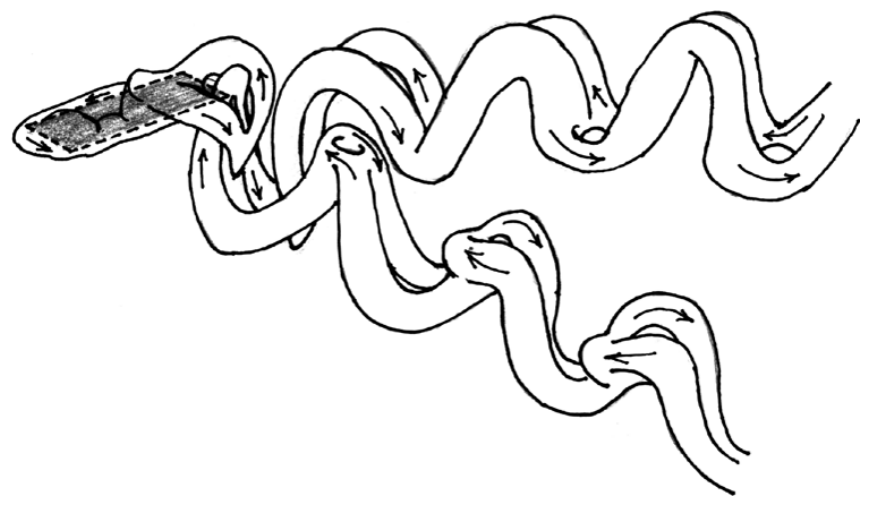

Fig. 6. Vortical patterns in a rectangular foil with aspect ratio 3, oscillating at Strouhal number 0.35 (from [150]). The patterns in the flow are isocontours of the $\lambda_{2}$ eigenvalue [153]

NACA 16-012 foil with aspect ratio 4. DeLaurier and Harris [17] report efficiencies in the range of $18-50 \%$ for a rectangular NACA 0012 flapping foil with aspect ratio of four, oscillating with heave amplitude equal to 0.625 chords at Reynolds number 30000 .

The presence of a leading edge vortex in three-dimensional foils depends strongly on the maximum angle of attack, and hence the specific load on the foil. The structure and connection of leading edge vorticity is a difficult subject, because flow visualization pictures are not typically clear. Maxworthy [82] had proposed that in three-dimensional wings leading edge vortices are helical vortices, which connect to the tip vortices. Numerical simulations in the wing of a hovering insect [95], [73] show a similar structure. This provides for a vorticity shedding mechanism different than in two-dimensional foils, since a helical vortex need not separate to convect downstream. Freymuth [33] shows pictures for low aspect ratio foils under high angle of attack, where both leading and trailing edge vorticity forms. The trailing edge vortices are connected back to the foil edges with alternating sign tip vortices, which appear at some point to cancel each other. This leads to the formation of vortex ring-like structures. The leading edge vortices also form rings but the flow is much more confused. Hence, it is possible to both have a direct connection between the leading edge vortex and the tip vortices, and have leading edge vortex shedding.

Leading edge vortices are an intricate part of flapping foil vorticity above a threshold angle of attack and Strouhal number. In a three-dimensional foil these leading edge vortices appear to be interconnected at the ends with bound vorticity and with shed vorticity.

A study of the qualitative and quantitative effect of the leading vortices as their strength increases from the mild values associated with enhance efficiency by Anderson et al. [6] to the very intense values found in insect flight [25] has not been performed yet. Such a study will provide a bridge in out understanding between the properties of fins for swimming to those of fins for flight.

1) Three-Dimensional Foils in Combinations of Feathering, Rowing, and Flapping Motion: In a series of papers Kato [52], [53], [54] has considered the forces generated by a foil with aspect ratio of order 1 - the aspect ratio found typically in the 
pectoral fins of fishes [126]. The fins performed three types of motion (see also Fig. 1):

1) rowing, i.e., forward-backward motion;

2) feathering, i.e., a twisting (or pitching, as defined in Fig. 1) motion about the axis of the fin;

3) flapping motion, i.e., rolling motion (inducing a heavelike motion, as shown in Fig. 1) about the root attachment of the fin transversely to the flow, when a steady stream exists.

The basic conclusion is that the propulsive efficiency of feathering or flapping foils (lift-based) is larger than the efficiency of drag-based rowing foils, in agreement with Walker and Westneat [128] who show a maximum efficiency of $10 \%$ for dragbased, contrasted with about $60 \%$ maximum efficiency for liftbased propulsion. Rowing is better suited for force generation in the absence of forward speed, however, providing better maneuverability, since it produces substantial thrust but small transverse forces. Also, it is found that a nonsinusoidal feathering motion combined with a sinusoidal rowing motion produces thrust accompanied with small transverse forces. Maximum efficiencies of the order of $45 \%$ are reported for the lift-based mode of propulsion Kato [55], [56].

\section{Multiple Foils and Foils Interacting With Bodies}

When two or more foils operate side by side, or foils operate near a wall or are attached to a vehicle, there are important interaction effects taking place. For example, two foils side by side may have strong vortex-to-vortex interaction effects resulting in a drag wake and serious deterioration of performance. Likewise, interaction with bodies can have similar effects.

Bandyopadhyay et al. [10] study a streamlined vehicle equipped with two flapping foils in close proximity. Force and efficiency measurements and flow visualization results, show strong interaction effects that require more parameters than the ones used for single foils.

Flow visualization in two side-by-side foils shows, similarly, that when foils oscillate very close to each other, a strong dragwake-like flow develops between the foils causing efficiency deterioration. Experiments with two foils flapping in antiphase, to emulate the Weis-Fogh mechanism [121], show that such strong interaction can produce sufficient thrust to propel a ship.

Dual foils have also been tested for efficiency. One of the issues in dual foils is the strong interaction between the wakes of the foils, which can take many forms:

- wakes can collapse into a single wake;

- wakes interact strongly forming two jets divided by a backflow region, which can deteriorate performance seriously;

- foils can be well separated, providing good thrust performance.

When foils flap against a body, or against a second foil, the conditions of the Weis-Fogh mechanism apply. Large forces are produced but these include large drag forces, while the resulting vortical patterns usually are different from single foils. In Tsutahara and Kimura [121] the Weis-Fogh mechanism is used to produce thrust for ship propulsion. Two rectangular plates with aspect ratio 1.8 were used up to Reynolds number 300000 . The efficiency was up to $58 \%$ for angular amplitude of $15^{\circ}$, lower for other conditions. Bandyopadhyay et al. [9], [10] employed two foils flapping against a middle flat plate. They report efficiencies up to $30 \%$, while the vortical patterns form a rapidly expanding wake.

\section{Foils Interacting With Oncoming Unsteady Flows}

Foils will invariably operate in environments that contain unsteady flow such as waves near the surface of the ocean; and large vortical structures and/or turbulence when operating in the wake of a propelled body, or in the wake of another foil or propeller. Several foils interacting with each other, a foil in the wake of sharply maneuvering object, foils operating with a turbulent ocean are only a few examples.

The study of the interaction between the wake of an upstream body and a downstream foil, the study of the interaction between foils in cascade, and foils in turbulent flow, require an understanding on how externally generated vorticity interacts with foil generated vorticity. Hence, multiple foils interacting with each other; and foils in the wake of upstream bodies, subject to organized shed vorticity or wake turbulence, require an understanding of how such vortical interactions affect the performance of the foils. This information can be valuable, since it can be used to preserve performance through sensing and closed loop control; and to study under what conditions - and how-such interactions can be used to actually improve propulsive performance [40].

Sparenberg and Wiersma [112], Koochesfahani and Dimotakis [63], Gopalkrishnan et al. [40], Streitlien et al. [115], and Beal et al. [11] have performed theoretical and experimental studies on the interaction of foils with upstream vorticity. Gopalkrishnan et al. [40] identified three modes of interaction.

- Upstream vortices are repositioned and then interact destructively with foil-generated vortices of the opposite sign to create a field of weak vortices (destructive mode), resulting in substantial increase of efficiency.

- Upstream vortices are repositioned and then join foil-generated vortices of the same sign to create a field of vigorous vortices (constructive mode), resulting in increase of thrust at the expense of reduced efficiency.

- Upstream vortices are repositioned and then pair with foilgenerated vortices of the opposite sign to create a field of vortex pairs, appearing in visualization as "mushroomstructures," resulting in a wide wake (pairing mode), resulting in a great variety of responses depending on the timing of vortex pairing.

Further work by Anderson [3] showed that there is one more dimension to the problem: leading edge vorticity can interact early with oncoming vorticity, resulting in patterns that resemble qualitatively the three major patterns of Gopalkrishnan et al. [40] but differ in several aspects of the flow especially close to the foil, hence affecting performance.

\section{E. Maneuvering Foils}

Flapping foils that are used to generate forces for maneuvering must either provide a steady lift force, often in addition to thrust, or provide a short-lived, high-magnitude force. There 
is close connection between flapping foils used for propulsion and those used for maneuvering since both depends on unsteady flow mechanisms to develop forces. The details differ, however, and hence the physical mechanisms and properties have differences as well.

Experiments to study the development of transient forces are relatively few. They contain cases of foils performing a transient motion [2], [98], [80]; foils performing a flapping motion with a superimposed bias angle to develop steady lift forces [88], [89], [98]; and combination of rowing, plunging, and feathering motions with bias angles to develop nonsinusoidally varying lift forces that can be used for positioning and maneuvering [52], [54], [55], [56], [79].

Hertel [46] and Ahlborn et al. [2] showed that a flapping foil develops a pair or pairs of interconnected vortices (which appear like rings in a three-dimensional view) when starting from a position of rest and performing a complete cycle of heave or pitch motion. Drucker and Lauder [20], [21] show the formation of sequences of inclined, interconnected ring-like structures in the wake of flapping pectoral fins of live fish.

As reported in Ohmi et al. [89], the bias angle in a pitching foil plays a significant role in determining the flow patterns up to a threshold Strouhal number-in the nomenclature of the authors, instead of Strouhal number, they use the product of reduced frequency and angle of oscillation.

In [98] and [80] a bias angle is used to produce steady lift in unsteadily flapping foils. Significant steady and unsteady lift, which is much higher, up to an order of magnitude, than under steady conditions, can be produced. The moderate aspect ratio, three-dimensional foil in Martin et al. [80] produced steady and unsteady lift forces comparable to those experienced by the two-dimensional foil employed by Read et al. [98]. This demonstrates once more that end-effects are less important in unsteady foils than steady foil, in accordance with the findings in [51], [14], and [25].

\section{F. The Influence of Cavitation on Foil Performance}

While cavitation in steadily moving foils is understood [5], this cannot be said for unsteady foils, where vortices form close to the foil, migrate in its wake, and interact with each other. The interaction among the tip vortices is of the destructive type, since the angle of attack is oscillatory-which explains why reduced frequency has often a beneficial effect, i.e., reducing cavitation.

Cavitation in unsteadily moving foils is known to be influenced by the reduced frequency of oscillation, but the information is very sparse and restricted to mostly visual observation [5], [45]. The vortical structure around the foil affects the cavitation properties significantly, so such an investigation must follow immediately after the investigation on the structure of the flow around and behind foils. First, the optimal range of foil operation must be investigated, i.e., with moderate angles of attack and Strouhal number; followed by larger angles of attack.

\section{G. Effect of Geometry and Flexing Stiffness of Foils}

Fish fins present great variability in shape, aspect ratio and structure, depending on the application they are intended for. Pectoral fins, for example, may have the shape of moderate- or low-aspect ratio foils for some fish; while for others, such as whales, large aspect ratio foils are used. Also, the flexibility of the foils ranges greatly. Foils can flex along their chord and/or along their span. Fish certainly employ passive flexibility and possibly actively controlled flexibility.

Kemp et al. [141] report experiments with a low-aspect ratio pitching foil, whose propulsive efficiency may double when the flexibility of the material is optimized. The material used in this study is relatively soft, allowing substantial flexing; hence the mechanism involved is that of resonance, i.e., added-mass energy storage and release within a cycle. Similar use of springlike materials has been shown to improve efficiency in theoretical studies [142] and in observations in animals [143]. The fact that low-aspect ratio wings can develop adequate efficiency is due to the alternating sign of the tip vortices, which reduce the "downwash" substantially, as noted earlier by Karpouzian et al. [51].

In a recent paper, Prempraneerach et al. [144] show that efficiency in high aspect ratio foils can increase substantially, exceeding $80 \%$, with small effect on the thrust, when chordwise flexibility is optimized. The material used is relatively stiff, causing a camber of the order of a few percent of the chord. A detailed parametric study shows that an optimal value of nondimensionalized elastic stiffness can be selected, which also provides a scaling law for chordwise flexible foils.

The significant improvement in propulsive efficiency through properly selected flexibility provides an explanation for the fact that many fish fins are flexible, more so than in birds, where lift production is more important than efficient thrust generation.

\section{REFERENCES}

[1] M. Acharya and M. H. Metwally, "Unsteady pressure field and vorticity production over a pitching foil," AIAA J., vol. 30, pp. 403-411, 1992.

[2] B. Ahlborn, D. Harper, R. Blake, D. Ahlborn, and M. Cam, "Fish without footprints," J. Theor. Boil., vol. 148, pp. 521-533, 1991.

[3] J. M. Anderson, "Vortex Control for Efficient Propulsion," Ph.D. dissertation, Joint Program, Massachusetts Inst. Technology and Woods Hole Oceanographic Inst., Cambridge, MA, 1996.

[4] R. E. A. Arndt, "Cavitation in fluid machinery and hydraulic structures," Ann. Rev. Fluid Mech., vol. 13, pp. 273-28, 1981

[5] —_ "Cavitation in vortical flows," Ann. Rev. Fluid Mech., vol. 34, pp. $143-175,2002$.

[6] J. M. Anderson, K. Streitlien, D. S. Barrett, and M. S. Triantafyllou, "Oscillating foils of high propulsive efficiency," J. Fluid Mech., vol. 360, pp. 41-72, 1998

[7] N. Bose and Y. Lien, "Propulsion of a fin whale (Balaenoptera physalus): Why the fin whale is a fast swimmer," in Proc. Roy. Soc. Lond., vol. 237, 1989, pp. 175-200.

[8] P. R. Bandyopadhyay, J. M. Castano, J. Q. Rice, R. B. Philips, W. H. Nedderman, and W. K. Macy, "Low speed maneuvering hydrodynamics of fish and small underwater vehicles," J. Fluids Eng., vol. 119, pp. 136-119, 1997a.

[9] P. R. Bandyopadhyay, M. J. Donnelly, W. H. Nedderman, and J. M. Castano, "A dual flapping foil maneuvering device for low-speed rigid bodies," in 3rd Int. Symp. Performance Enhancement for Marine Vehicles, Newport, RI, 1997b.

[10] P. R. Bandyopadhyay, J. M. Castano, M. J. Donnelly, W. H. Nedderman, and M. J. Donnelly, "Experimental simulation of fish-inspired unsteady vortex dynamics on a rigid cylinder," J. Fluids Eng., vol. 122, pp. 219-238, 2000.

[11] D. N. Beal, F. S. Hover, and M. S. Triantafyllou, "The effect on thrust and efficiency of an upstream Karman wake on an oscillating foil," in Proc. UUST, Durham, NH, 2001.

[12] C. E. Brown and W. H. Michael, "Effect of leading edge separation on the lift of delta wing," J. Aerosp. Sci., vol. 21, p. 690, 1954.

[13] H. K. Cheng and L. E. Murillo, "Lunate-tail swimming propulsion as a problem of curved lifting line in unsteady flow. Part 1 . Asymptotic theory," J. Fluid Mech., vol. 143, pp. 327-350, 1984. 
[14] J.-Y. Cheng, L.-X. Zhuang, and B.-G. Tong, "Analysis of swimming three-dimensional waving plates," J. Fluid Mech., vol. 232, pp. 341-355, 1991.

[15] C. Y. Chow, C. L. Chen, and M. K. Huang, "Trapping of free vortex by airfoils with surface suction," AIAA J., vol. 24, p. 1217, 1985.

[16] J. Cox, "The revolutionary Kasper wing," AIAA Soaring, Dec. 20, 1973.

[17] J. D. DeLaurier and J. M. Harris, "Experimental study of oscillating wing propulsion," J. Aircraft, vol. 19, no. 5, pp. 368-373, 1982.

[18] M. H. Dickinson, "The effect of wing rotation on unsteady aerodynamic performance at low Reynolds numbers," J. Exp. Biol., vol. 192, pp. 179-206, 1994.

[19] M. H. Dickinson, F. O. Lehmann, and S. P. Sane, "Wing rotation and the aerodynamic basis of insect flight," Science, vol. 284, pp. 1954-1960, 1999.

[20] E. G. Drucker and G. V. Lauder, "Locomotor forces on a swimming fish: Three-dimensional vortex wake dynamics quantified using DPIV," J. Exp. Biol., vol. 202, pp. 2393-2412, 1999.

[21] — "A hydrodynamic analysis of fish swimming speed: Wake structure and locomotor force in slow and fast labriform swimmers," J. Exp. Biol., vol. 203, pp. 2379-2393, 2000.

[22] — - "Locomotor function of the dorsal fin in teleost fishes: Experimental analysis of the wake forces in sunfish," J. Exp. Biol., vol. 204, pp. 2943-2958, 2001.

[23] C. P. Ellington, "The aerodynamics of hovering insect flight," Phil. Trans. Roy. Soc., ser. B, vol. 305, pp. 17-181, 1984.

[24] C. P. Ellington, C. Vanderberg, A. Wilmott, and A. Thomas, "Leading edge vortices in insect flight," Nature, vol. 384, pp. 626-630, 1996.

[25] C. P. Ellington, "The novel aerodynamics of insect flight: Applications to micro-air vehicles," J. Exp. Biol., vol. 202, pp. 3439-3448, 1999.

[26] F. E. Fish, "Biological designs for enhanced maneuverability: Analysis of marine mammal performance," in 10th Int. Symp. Unmanned Untethered Submersible Technology, Special Session on Bio-Engineering Research Related to AUV, Durham, NH, 1997, pp. 109-117.

[27] F. E. Fish and C. A. Hui, "Dolphin swimming-A review," Mammal Rev., vol. 21, pp. 181-195, 1991.

[28] F. E. Fish, "Influence of hydrodynamic design and propulsive mode on mammalian energetics," Aust. J. Zool., vol. 42, pp. 79-101, 1993.

[29] — "Transitions from drag-based to lift-based propulsion in mammalian swimming," Amer. Zool., vol. 36, pp. 628-641, 1996.

[30] P. Freymuth, "Propulsive vortical signature of plunging and pitching airfoils," AIAA J., vol. 26, pp. 881-883, 1988.

[31] — "Visualizing the connectivity of vortex systems for pitching wings," J. Fluids Eng., vol. 111, pp. 217-220, 1989.

[32] _ "Thrust generation by an airfoil in Hover modes," Exp. Fluids, vol. 9, pp. 17-24, 1990.

[33] - "Physical vortex visualization as a reference for computer simulation," in Vortex Methods and Vortex Motion, K. E. Gustafson and J. A. Sethian, Eds. Philadelphia, PA: SIAM, 1991, pp. 65-94.

[34] H. R. Frith and R. W. Blake, "Mechanical power output and hydromechanical efficiency of northern pike (Esox Lucius) fast-starts," J. Exp. Biol., vol. 198, pp. 1863-1873, 1995.

[35] S. B. Furber and J. E. F. Williams, "Is the Weis-Fogh principle exploitable in turbomachinery?," J. Fluid Mech., vol. 94, no. 3, pp. 519-540, 1979.

[36] M. Gad-el-Hak and C. M. Ho, "Unsteady vortical flow around threedimensional lifting surfaces," AIAA J., vol. 24, pp. 713-721, 1980.

[37] I. E. Garrick, "Propulsion of a Flapping and Oscillating Foil," NACA, Technical Report 567, 1936.

[38] M. Gharib, E. Rambod, D. Dabiri, and M. Hammache, "Pulsatile heart flow: A universal time scale," in Proc. 2nd Int. Conf. Experimental Fluid Mech., M. Onorato, Ed., Torino, Italy, 1994.

[39] M. Gharib, E. Rambod, and K. Shariff, "A universal time scale for vortex ring formation,” J. Fluid Mech., vol. 360, pp. 121-140, 1998.

[40] R. Gopalkrishnan, M. S. Triantafyllou, G. S. Triantafyllou, and D. S. Barrett, "Active vorticity control in a shear flow using a flapping foil," J. Fluid Mech., vol. 274, pp. 1-21, 1994.

[41] J. H. Greydanus, A. I. Van de Vooren, and H. Bergh, "Experimental determination of the aerodynamic coefficients of an oscillating wing in incompressible, two-dimensional flow," National Luchtvaart-Laboratorium, Amsterdam, the Netherlands, pt. I-IV, Rep. F-101, 102, 103, and $104,1952$.

[42] I. Gursul, H. Lin, and C. M. Ho, "Vorticity dynamics of 2-D and 3-D wings in unsteady free stream,", Reno, NV, AIAA Paper 91-0010, 1991.

[43] I. Gursul and C. Ho, "High aerodynamic loads on an airfoil submerged in an unsteady stream," AIAA J., vol. 30, pp. 1117-1119, 1992.

[44] D. G. Harper and R. W. Blake, "Prey capture and the fast-start performance of northern pike (Esox Lucius)," J. Exp. Biol., vol. 155, pp. 175-192, 1991.
[45] D. P. Hart, A. Acosta, and A. Leonard, "Observations of cavitation and wake structure of unsteady tip vortex flows," in Proc. Intern. STG Symp. Propulsors and Cavitation, Hamburg, Germany, 1992, pp. 121-127.

[46] H. Hertel, Structure Form and Movement. New York: Reinhold, 1966.

[47] K. G. Hoppe, "The dynamo-elastic oscillating foil propeller," Schiff Hafen, vol. 5, pp. 54-61, 1989.

[48] F. S. Hover, D. R. Read, O. Haugsdal, and M. S. Triantafyllou, "Experiments with a 2D oscillating foil for propulsion and maneuvering," in Proc. UUST, Durham, NH, 2001.

[49] H. Isshiki and M. Murakami, "A theory of wave devouring propulsion," J. Soc. Nav. Arch., vol. 156, pp. 102-114, 1984.

[50] K. D. Jones, C. M. Dohring, and M. F. Platzer, "Experimental and computational investigation of the Knoller-Betz effect," AIAA J., vol. 36, no. 7, pp. 1240-1246, 1998.

[51] G. Karpouzian, G. Spedding, and H. K. Cheng, "Lunate-tail swimming propulsion. Part 2. Performance analysis," J. Fluid Mech., vol. 210, pp. 329-351, 1990.

[52] N. Kato, "Locomotion by mechanical pectoral fins," J. Mar. Sci. Technol., vol. 3, pp. 113-121, 1998.

[53] N. Kato and T. Inaba, "Hovering performance of fish robot with apparatus o pectoral fin motion," in 10th Int. Symp. Unmanned Untethered Submersible Technology, 1997.

[54] N. Kato, "Control performance of fish robot with mechanical pectoral fins in horizontal plane," IEEE J. Oceanic Eng., vol. 25, no. 1, pp. 121-129, 2000.

[55] — - "Hydrodynamic characteristics of mechanical pectoral fin," ASME J. Fluids Eng., vol. 121, pp. 605-613, 1999.

[56] — "Three-motor-driven mechanical pectoral fin," in Proc. 11th Intern. Symp. Unmanned Untethered Submersible Technology, Durham, NH, 1999, pp. 467-476.

[57] J. Katz and D. Weihs, "Hydrodynamic propulsion by large amplitude oscillation of an airfoil with chordwise flexibility," J. Fluid Mech., vol. 88, pp. 485-497, 1978.

[58] - "Behavior of vortex wakes from oscillating airfoils," AIAA J., vol. 15, pp. 861-863, 1978.

[59] _ , "Large amplitude unsteady motion of a flexible slender propulsor," J. Fluid Mech., vol. 90, pp. 713-723, 1979.

[60] R. Katzmayr, "Effect of Periodic Changes of Angle of Attack on Behavior of Airfoils,", NACA Technical Memorandum, TM-147, 1922.

[61] A. B. Kessel, "Aerodynamic characteristics of dragonfly wing sections compared with technical airfoils," J. Exp. Biol., vol. 203, pp. 3125-3135, 2000.

[62] M. Koochesfahani, "Vortical patterns in the wake of an oscillating foil," AIAA J., vol. 27, pp. 1200-1205, 1989.

[63] M. Koochesfahani and P. Dimotakis, "A Cancellation Experiment in a Forced Turbulent Shear Layer,", AIAA Technical Paper 88-3713-CP, 1988.

[64] I. Kroo, "Drag due to lift: Concepts for prediction and reduction," Ann. Rev. Fluid Mech., vol. 33, pp. 587-617, 2001.

[65] E. W. Krupa, "A Wind Tunnel Investigation of the Kasper Wing Concept,", AIAA Technical Paper 77-310, 1977.

[66] J. Kumph and M. S. Triantafyllou, "A fast-starting and maneuvering vehicle, the Robopike," in Proc. Int. Symp. Seawater Drag Reduction, Newport, RI, 1998.

[67] P. S. K. Lai, N. Bose, and R. C. McGregor, "Wave propulsion from a flexible-armed, rigid-foil propulsor," Marine Technology, vol. 30, no. 1, pp. 28-36, 1993.

[68] J. C. S. Lai and M. F. Platzer, "The jet characteristics of a plunging airfoil," in 36th AIAA Aerospace Sciences Meeting, Paper AIAA-98-0101, Reno, NV, 1998.

[69] G. V. Lauder, "Function of the caudal fin during locomotion in fishes: Kinematics, flow visualization, and evolutionary patterns," Amer. Zool., vol. 40, pp. 101-122, 2000.

[70] J. Lighthill, "HydroMech. of aquatic animal propulsion," Annu. Rev. Fluid Mech., vol. 1, pp. 413-445, 1969.

[71] _ "Aquatic animal propulsion of high hydromechanical efficiency," J. Fluid Mech., vol. 44, pp. 265-301, 1970.

[72] —, Mathematical Biofluiddynamics. Philadelphia, PA: SIAM, 1975.

[73] H. Liu and K. Kawachi, "A numerical study of insect flight," J. Comput. Phys., vol. 146, pp. 124-156, 1998.

[74] G. V. Longvinovich, "Hydrodynamics of a thin, flexible body," in $\mathrm{Hy}$ drodynamic Problems in Bionics, Washington, DC, Mar. 1971, pp. 4-11. Joint Publications Research Service-52605.

[75] M. W. Luttges, C. Somps, M. Kliss, and M. C. Robinson, "Unsteady separated flows: Generation and use by insects," in Workshop on Unsteady Separated Flow, CO, Aug. 1983. 
[76] L. Luznik and N. Bose, "Propulsive thrust of an oscillating foil at large angles of attack: Experimental study," in Proc. Amer. Towing Tank Conf., Iowa, 1998.

[77] W. J. MacCroskey, "Unsteady airfoils," Ann. Rev. Fluid Mech., vol. 14, pp. 285-311, 1982.

[78] C. Maresca, D. Favier, and J. Rebont, "Experiments on an airfoil at high angle of incidence in longitudinal oscillations," J. Fluid Mech., vol. 92, pp. 671-690, 1979 .

[79] C. B. Martin, "Propulsive Performance of a Rolling and Pitching Wing," M.S. Thesis, Massachusetts Institute of Technology, 2001.

[80] C. B. Martin, F. S. Hover, and M. S. Triantafyllou, "Propulsive performance of a rolling and pitching wing," in Proc. UUST, Durham, NH, 2001

[81] T. Maxworthy, "Experiments on the Weis-Fogh mechanism of lift generation by insects in hovering flight. Part I. Dynamics of the fling," $J$. Fluid Mech., vol. 93, pp. 47-63, 1979.

[82] — , "The fluid dynamics of insect flight," Ann. Rev. Fluid Mech., vol. 13, pp. 329-350, 1981.

[83] J. E. McCune and T. S. Tavares, "Perspective: Unsteady wing theory-The Kármán/Sears legacy," J. of Fluids Engineering, vol. 115, no. 4, pp. 548-560, 1993.

[84] U. B. Mehta and Z. Lavan, "Starting vortex, separation bubbles and stall: A numerical study of laminar unsteady flow around an airfoil," J. Fluid Mech., vol. 67, pp. 227-256, 1975.

[85] U. Mueller, B. van den Heuvel, E. Stamhuis, and J. Videler, "Fish foot prints: Morphology and energetics of the wake behind a continuously swimming mullet (Chelon Labrosus Risso)," J. Exp. Biol., vol. 200, pp. 2893-2806, 1997

[86] J. C. Nauen and G. V. Lauder, "Hydrodynamics of caudal fin locomotion by chub mackerel Scomber japonicus (Scombridae)," J. Exp. Biol., vol. 205, pp. 1709-1724, 2002

[87] H. Ohashi and N. Ishikawa, "Visualization study of flow near the trailing edge of an oscillating airfoil," Bull. Jap. Soc. Mech. Engin., vol. 15, no. 85, pp. 840-847, 1972.

[88] K. Ohmi, M. Coutanceau, T. P. Loc, and A. Dulieu, "Vortex formation around an oscillating and translating airfoil at large incidences," J. Fluid Mech., vol. 211, pp. 37-60, 1990.

[89] K. Ohmi, M. Coutanceau, O. Daube, and T. P. Loc, "Further experiments on vortex formation around an oscillating and translating airfoil at large incidences," J. Fluid Mech., vol. 225, pp. 607-630, 1991.

[90] Y. Oshima and K. Oshima, "Vortical flow behind an oscillating foil," in Proc. 15th Int. Congr. Amsterdam, the Netherlands, 1980, pp. 357-368.

[91] Y. Oshima and A. Natsume, "Flow field around an oscillating foil," in Proc. 2nd Int. Symp. Flow Visualization Flow Visualization II, W. Merzkirch, Ed., Bochum, Germany, 1980, pp. 295-299.

[92] S. O. Park, J. S. Kim, and B. I. Lee, "Hot-wire meausurements of near wakes behind an oscillating airfoil," AIAA J., vol. 28, pp. 22-28, 1990.

[93] D. R. Poling and D. P. Telionis, "The trailing edge of a pitching airfoil at high reduced frequencies," J. Fluids Eng., vol. 109, pp. 410-414, 1987.

[94] R. Ramamurti and W. C. Sandberg, "Simulation of flow about flapping airfoils using a finite element incompressible flow solver," AIAA J., vol. 39, pp. 253-260, 2001.

[95] _ "A three-dimensional computational study of the aerodynamic mechanisms of insect flight," J. Exp. Biol., vol. 205, pp. 1507-1518, 2002.

[96] R. Ramamurti, W. C. Sandberg, R. Lohner, J. Walker, and M. W. Westneat, "Fluid dynamics of flapping aquatic flight in the bird wrasse: Three-dimensional unsteady computations with fin deformations," $J$. Exp. Biol., vol. 205, pp. 2997-3008, 2002.

[97] J. M. V. Rayner, "Dynamics of the vortex wakes of flying and swimming vertebrates," in Biological Fluid Dynamics, C. P. Ellington and T. J. Pedley, Eds. Cambridge, U.K.: Society of Experimental Biology, 1995, pp. 131-155.

[98] D. A. Read, F. S. Hover, and M. S. Triantafyllou, "Forces on oscillating foils for propulsion and maneuvering," J. Fluids Struct., vol. 17, pp. 163-183, 2003.

[99] W. C. Reynolds and L. W. Carr, "Review of unsteady, driven, separated flows,", AIAA Paper 85-0527, 1985.

[100] D. Rockwell, "Vortex-body interactions," Annu. Rev. Fluid Mech., vol. 30, pp. 199-229, 1998.

[101] J. J. Rohr, E. W. Hendricks, L. Quigley, F. E. Fish, J. W. Gipatrick, and J. Scardina-Ludwig, "Observations of dolphin swimming speed and strouhal number,", San Diego, CA, SPAWAR Tech. Rep. 1769, 1998.

[102] M. Rosenfeld, M. Gharib, and E. Rambod, "Circular and formation number of laminar vortex rings," J. Fluid Mech., vol. 376, pp. 297-18, 1998.

[103] V. J. Rossow, "Lift enhancement by an externally trapped vortex," $J$. Aircraft, vol. 15, p. 618, 1977.
[104] P. G. Saffman and J. S. Sheffield, "Flow over a wing with an attached free vortex," Stud. Appl. Math., vol. 57, p. 107, 1977.

[105] W. Sandberg and R. Ramamurti, "Unsteady flow computations for oscillating fins: A status report," in Proc. 11th Int. Symp. Unmaned Untethered Submersible Technology, Durham, NH, 1999.

[106] S. P. Sane and M. H. Dickinson, "The control of flight force by a flapping wing: Lift and drag production," J. Exp. Biol., vol. 204, pp. 2607-2626, 2001

[107] — "The aerodynamic effects of wing rotation and a revised quasisteady model of flapping flight," J. Exp. Biol., vol. 205, pp. 1087-1096, 2002.

[108] B. Satyanarayana and S. Davis, "Experimental studies of unsteady trailing-edge conditions," AIAA. J., vol. 16, pp. 125-129, 1978.

[109] J. O. Scherer, Experimental and Theoretical Investigation of Large Amplitude Oscillating Foil Propulsion Systems: U.S. Army Engineering Research and Development Laboratories, 1968.

[110] S. N. Singh and P. R. Bandyopadhyay, "A theoretical control study of the biologically inspired maneuvering of a small vehicle under a free surface wave,", Newport, RI, NUWC-NPT Tech. Rep. 10 816, 1997.

[111] M. J. C. Smith, P. J. Wilkin, and M. H. Williams, "The advantages of an unsteady panel method in modeling the aerodynamic forces on rigid flapping wings," J. Exp. Biol., vol. 199, pp. 1073-1083, 1996.

[112] J. A. Sparenberg and A. K. Wiersma, "On the efficiency increasing interaction of thrust producing lifting surfaces," in Swimming and Flying in Nature, T. Wu, C. J. Brokaw, and C. Brennen, Eds. New York: Plenum, 1975, vol. 2, pp. 891-917.

[113] E. Stamhuis and J. Videler, "Quantitative flow analysis around aquatic animals using laser sheet particle image velocimetry," J. Exp. Biol., vol. 198, pp. 283-294, 1995

[114] K. Streitlien and M. S. Triantafyllou, "Force and moment on a Joukowski profile in the presence of point vortices," AIAA J., vol. 33 , no. 4 , pp. 603-610, 1995

[115] K. Streitlien, G. S. Triantafyllou, and M. S. Triantafyllou, "Efficient foil propulsion through vortex control," AIAA J., vol. 34, no. 11, pp. 2315-2319, 1996

[116] M. Sun and J. Tang, "Unsteady aerodynamic force generation by a model fruit fly wing in flapping motion," J. Exp. Biol., vol. 205, pp. 55-70, 2002a.

[117] — , "Lift and power requirements of hovering flight in Drosophila virilis," J. Exp. Biol., vol. 205, pp. 2413-2427, 2002b.

[118] S. Taneda, "Visual study of unsteady separated flows around bodies," Progr. Aerosp. Sci., vol. 17, pp. 287-48, 1977.

[119] M. S. Triantafyllou, G. S. Triantafyllou, and R. Gopalkrishnan, "Wake mech. for thrust generation in oscillating foils," Phys. Fluids A, vol. 3 , no. 12 , pp. $2835-2837,1991$.

[120] G. S. Triantafyllou, M. S. Triantafyllou, and M. A. Grosenbaugh, "Optimal thrust development in oscillating foils with application to fish propulsion,” J. Fluids Struct., vol. 7, pp. 205-224, 1993.

[121] M. Tsuhara and T. Kimura, "An application of the Weis-Fogh mechanism to ship propulsion," J. Fluids Eng., vol. 109, pp. 107-113, 1987.

[122] J. R. Usherwood and C. P. Ellington, "The aerodynamics of revolving wings," J. Exp. Biol., vol. 205, pp. 1547-1576, 2002.

[123] J. Videler, Fish Swimming. London, U.K.: Chapman and Hall, 1993.

[124] M. R. Visball and J. S. Shang, "Investigation of the flow structure around a rapidly pitching foil," AIAA J., vol. 27, no. 8, pp. 1044-1051, 1989.

[125] J. A. Walker, "Rotational lift: Something different or more of the same?," J. Exp. Biol., vol. 205, pp. 3783-3792, 2003.

[126] J. A. Walker and M. W. Westneat, "Labriform propulsion in fishes: Kinematics of flapping aquatic flight in the bird wrasse Gomphosus Varius (Labridae)," J. Exp. Biol., vol. 200, pp. 1549-1569, 1997.

[127] J. A. Walker, "Does a rigid body limit maneuverability?," J. Exp. Biol., vol. 203, pp. 3391-3396, 2000.

[128] J. A. Walker and M. W. Westneat, "Mechanical performance of aquatic rowing and flying," in Proc. Roy. Soc. Lond., vol. B 267, 2000, pp $1875-1881$.

[129] — , "Performance limits of labriform propulsion and correlates with fin shape and motion," J. Exp. Biol., vol. 205, pp. 177-187, 2002

[130] J. A. Walker, "Rotational lift: Something different or more of the same?," J. Exp. Biol., vol. 205, pp. 3783-3781, 2002.

[131] J. Wang, "Vortex shedding and frequency selection in flapping flight," $J$. Fluid Mech., vol. 410, pp. 323-341, 2000

[132] I. Watanabe and S. Ishida, "An experiment of oscillating hydrofoil in waves," in Engineering Science, Fluid Dynamics, A Symposium to Honor T. Y. Wu, G. T. Yates, Ed.. Singapore, 1990, pp. 331-340.

[133] T. Weis-Fogh, "Quick estimates of flight fitness in hovering animals including novel mechanisms for lift production," J. Exp. Biol., vol. 59 , pp. 169-230, 1973. 
[134] H. Werle and M. Gallon, "Study by hydrodynamic visualizations of various processes for controlling separated flows," La Rech. Aero., vol. 2, pp. 70-75, 1976.

[135] M. J. Wolfgang, J. M. Anderson, M. A. Grosenbaugh, D. K. P. Yue, and M. S. Triantafyllou, "Near-body flow dynamics in swimming fish," $J$. Exp. Biol., vol. 202, pp. 2303-2327, 1999.

[136] J. Z. Wu, A. D. Vakili, and J. M. Wu, "Review of the physics of enhancing vortex lift by unsteady excitation," Prog. Aerosp. Sci., vol. 28, pp. 73-131, 1991.

[137] T. Wu, "Swimming of a waving plate," J. Fluid Mech., vol. 10, pp. 321-344, 1961.

[138] - "Hydromechanics of swimming propulsion," J. Fluid Mech., vol. 46, pp. 337-355, 1971.

[139] I. Yamamoto, Y. Terada, T. Nagamatu, and Y. Imaizumi, "Propulsion system with flexible/rigid oscillating fin," IEEE J. Oceanic Eng., vol. 20, pp. 23-30, 1995.

[140] K. D. von Ellenrieder, K. Parker, and J. Soria, "Flow structures behind a heaving and pitching finite-span wing," J. Fluid Mech., vol. 490, pp. 129-138, 2003

[141] M. Kemp, B. Hobson, and C. Pell, "Energetics of the oscillating fin thruster," in Proc. 13th Int. Symp. Unmanned Untethered Submersible Technology (UUST), Durham, NH, 2003.

[142] M. M. Murray and L. E. Howle, "Spring stiffness influence on an oscillating propulsor," J. Fluids Struct., vol. 17, pp. 915-926, 2003.

[143] S. L. Lindstedt, T. E. Reich, P. Keim, and P. C. LaStayo, "Review: Do muscles function as adaptable locomotor springs?," J. Exp. Biol., vol. 205, pp. 2211-2216, 2002.

[144] P. Prempraneerach, F. S. Hover, and M. S. Triantafyllou, "The effect of chordwise flexibility on the thrust and efficiency of a flapping foil," in 13th Int. Symp. Unmanned Untethered Submersible Techn., Durham, NH, Aug. 24-27, 2003.

[145] R. Motani, "Scaling effects in caudal fin propulsion and the speed of ichthyosaurs," Nature, vol. 415, pp. 309-312, 2002.

[146] G. K. Taylor, R. L. Nudds, and A. L. R. Thomas, "Flying and swimming animals cruise at a Strouhal number tuned for high power efficiency," Nature, vol. 425, pp. 707-711, 2003.

[147] J. C. Liao, D. N. Beal, G. V. Lauder, and M. S. Triantafyllou, "Fish exploiting vortices use less muscle," Science, vol. 302, pp. 1566-1569, 2003.

[148] P. W. Webb, "Designs for stability and maneuverability in aquatic vertebrates: What can we learn?," in Proc. 10th Int. Symp. Unmanned Untethered Submersible Technology, Durham, NH, 1997, pp. 86-108.

[149] — "Maneuverability versus stability? Do fish perform well in both?," in Proc. 1st Int. Symp. Aqua Bio-Mechanisms (ISABMEC 2000). Honolulu, HI, Aug. 2000.

[150] L. Guglielmini, "Modeling of Thrust Generating Foils," Ph.D. Thesis, University of Genova, Italy, 2004

[151] L. Guglielmini and P. Blondeaux, "Propulsive efficiency of oscillating foils," Eur. J. Mech. B Fluids, to be published.

[152] L. Guglielmini, P. Blondeaux, and G. Vittori, "A simple model of propulsive oscillating foils," Ocean Eng., to be published.

[153] J. Jeong and F. Hussein, "On the identification of a vortex," J. Fluid Mech., vol. 285, pp. 69-94, 1995.

[154] G. C. Lewin and H. Haj-Hariri, "Modeling thrust generation of a twodimensional heaving airfoil in a viscous flow," J. Fluid Mech., vol. 492, pp. 339-362, 2003.

[155] F. S. Hover, O. Haugsdal, and M. S. Triantafyllou, "Control of angle of attack profiles in flapping foil propulsion,” J. Fluids Struct., vol. 19, pp. $37-47,2003$

[156] J. J. Rohrs and F. E. Fish, "Strouhal numbers and optimization of swimming by odontocete cetaceans," J. Exp. Biol., vol. 207, pp. 1633-1642, 2004.

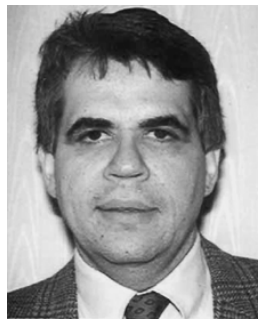

Michael S. Triantafyllou was born in Athens, Greece, in 1951. He received the diploma in naval architecture and marine engineering from the National Technical University of Athens in 1974 and the S.M. and Sc.D. degrees in ocean engineering from the Massachussetts Institute of Technology (MIT), Cambridge, in 1977 and 1979, respectively.

He has been Assistant Professor (1979-1983), Associate Professor (1983-1990), and Professor (1990-present) in the Department of Ocean Engineering, MIT. He has published in the areas of dynamics and control of marine systems, experimental fluid mechanics, and biomimetics. He was a Visiting Professor at ETH Zurich (1999), NTU Athens (1994 and 2000), NTH Norway (1993), and Kyushu University, Japan (1986).

Prof. Triantafyllou is a member of the Society of Naval Architects and Marine Engineers, the American Physical Society, and the International Society for Offshore and Polar Engineers. His awards include RoboTuna on permanent exhibit at the Museum of Science, London (since 1998); prototype RoboTuna in national traveling exhibit on robots, Science Museum of Minnessota (2003-2004); Discover Magazine Award for Technological Innovation (1998); ABS/Linnard Prize for Best Paper in the Transactions of SNAME (1997); Highlight Paper of 1995 Scientific American; and H. L. Doherty Professorship in Ocean Utilization (1983-1985).

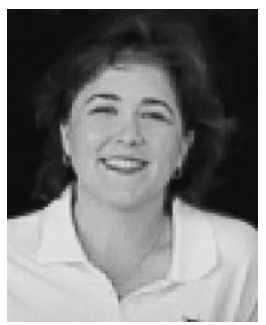

Alexandra H. Techet received the B.S.E. degree in mechanical and aerospace engineering from Princeton University, Princeton, NJ, in 1995 and the M.S. and Ph.D. degrees in oceanographic engineering from the joint program of the Massachusetts Institute of Technology (MIT), Cambridge, and Woods Hole Oceanographic Institute (WHOI), Woods Hole, MA, in 1998 and 2001, respectively.

She joined the Faculty of the Ocean Engineering Department, MIT, in 2002, as an Assistant Professor. She became the H. L. Doherty Professorship in Ocean Utilization (2002-2004). Her research interests include experimental marine hydrodynamics, ship breaking waves, fluid-structure interactions, and fish-like swimming and maneuvering.

Prof. Techet is a member of the American Society of Mechanical Engineers, American Physical Society, Marine Technology Society, and International Society of Offshore and Polar Engineers.

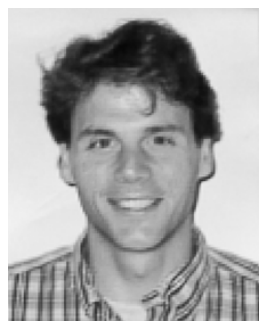

Franz S. Hover (A'93) received the B.S. degree in mechanical engineering from Ohio Northern University, Ada, in 1987 and the S.M. and Sc.D. degrees in oceanographic and mechanical engineering from the Woods Hole Oceanographic Institution/Massachusetts Institute of Technology (MIT), Cambridge, in 1989 and 1993, respectively.

He was a Postdoctoral Fellow with the Monterey Bay Aquarium Research Institute. He has been a regular Consultant to industry. He is currently a Principal Research Engineer in the Department of Ocean Engineering, MIT. His areas of research interest include design and applied control of marine systems.

Dr. Hover is a member of the American Society of Mechanical Engineers. 